\title{
Interessenkonflikte bei Guidelines und Choosing-wisely-Listen
}

\author{
Schweizerische Akademie der Medizinischen Wissenschaften (SAMW)
}

\section{Hintergrund}

Das Positionspapier "Nachhaltige Medizin» der SAMW fordert, dass die Auswahl medizinischer Interventionen mit Augenmass zu erfolgen hat. Die Haltung, «möglichst alles zu machen, und zwar möglichst sofort», solle ersetzt werden durch die Haltung, "genug zu machen, aber nicht zu viel». Das Positionspapier führt drei Bedingungen an, unter denen solche "wise choices» überhaupt erst möglich werden:

- «Unabhängige Guidelines definieren eine 'Baseline' für Qualität im weiten Sinn. Die therapeutische Freiheit ist weiterhin gewährleistet - allerdings mit der Einschränkung, dass ein Abweichen von einer Guideline begründet werden muss.

- Der Arzt und der Patient entscheiden gemeinsam im Sinne des 'shared decision making'; dies bedeutet auch, allfällig vorhandene Probleme (Alter, Komorbidität) anzusprechen und Unsicherheit zu teilen.

- Interessenkonflikte sind konsequent offenzulegen, und der Umgang damit ist zu klären. Dies gilt nicht nur für die Erstellung von Guidelines, sondern auch für die Ausgestaltung von Anreizsystemen» (Scheidegger et al. 2012).

In Fachkreisen wie in der breiteren Öffentlichkeit besteht ein zunehmendes Bewusstsein, dass Interessenkonflikte die Qualität und Fairness der medizinischen Versorgung unterminieren können. Zugleich wird das Recht der Patientinnen und Patienten auf zugängliche, evidenzbasierte Informationen als Grundlage für ein Shared Decision Making anerkannt (Gerber et al. 2016). Wenn Leitlinien und Choosing-wisely-Listen aufgrund von Interessenkonflikten verzerrte Informationen und Bewertungen enthalten, untergräbt dies den Patientennutzen. Auch angesichts steigender Gesundheitsausgaben und eines politischen Drucks zur Kostendämpfung darf davon ausgegangen werden, dass Guidelines und Choosing-wisely-Listen künftig verstärkte Beachtung zukommt. Umso wichtiger ist eine Entwicklung transparenter, qualitativ hochstehender Produkte gemäss etablierten internationalen Standards. Auf diese Weise kön- nen die Akzeptanz in der Ärzteschaft und die Glaubwürdigkeit in der Öffentlichkeit weiter positiv beeinflusst werden.

\section{Empfehlungen}

Guidelines sind aufwendig in der Entwicklung, können aber helfen, Prozesse zu optimieren, unbegründete Variationen in der medizinischen Praxis zu vermeiden und Behandlungsergebnisse zu verbessern (Rodney/ Feder 1998, Hostettler et al. 2014). Guidelines von mangelhafter Qualität bedeuten nicht nur eine Verschwendung von Ressourcen, sondern können schlimmstenfalls auch die Versorgung negativ beeinflussen. So heben Tools für das Assessment der Qualität von Guidelines auch den Umgang mit Interessenkonflikten ab. Das AGREE (Appraisal of Guidelines for Research and Education) II Instrument, welches auch für klinische Leitlinien geeignet ist, führt in der Domäne «Editorial independence» zwei Punkte auf: «There should be an explicit statement that the views or interests of the funding body have not influenced the final recommendation" und "There should be an explicit statement that all group members have declared whether they have any competing interests». Ein klar definierter, reflektierter Umgang mit Interessenkonflikten kann somit einen signifikanten Beitrag zur Qualität einer Guideline leisten.

\section{Definition "Interessenkonflikte»}

In Anlehnung an die Definition der IOM (Lo et al. 2009), die auf Thompson (1993) gründet, können Interessenkonflikte definiert werden als «Gegebenheiten, die ein Risiko dafür schaffen, dass professionelles Urteilsvermögen oder Handeln, welches sich auf ein primäres Interesse bezieht, durch ein sekundäres Interesse unangemessen beeinflusst wird» (Strech et al. 2011). Interessenbindungen können, müssen aber nicht zu Interessenkonflikten führen. Interessenkonflikte können das Individuum oder auch die Institution betreffen, für welche der Einzelne tätig ist («institutionelle Interessenkonflikte»). Vielfach geschieht der Einfluss von Interessenkonflikten auf das Urteilsvermögen unbewusst. Es liegt somit häufig keine bewusste Entscheidung vor, Sachverhalte zu verzerren oder zu vernachlässigen; es wird in diesem Sinne vom «bias blind spot» gesprochen (Lieb et al. 2011). Das Entscheidende am Konzept «bias blind spot» ist der Umstand, dass Personen mit Interessenkonflikt zu ihrem verzerrten Urteil gelangen, ohne es wahrzunehmen, und darüber hinaus die Verzerrung auch nicht erkennen, wenn sie darauf aufmerksam gemacht werden - sie "sehen" die Verzerrung tatsächlich nicht, daher die Metapher des blinden Flecks (Ehrlinger; Gilovich; Ross, 2005). Dabei ist allerdings zu berücksichtigen, dass Interessenbindungen (z.B. ein finanzieller Vorteil) sich auf Personen unterschiedlich auswirken können und die Vulnerabilität für Verzerrungen des Urteils variieren kann.

Interessenkonflikte müssen nicht unbedingt finanzieller Natur sein. Auch immaterielle Interessenkonflikte (Beteiligung an Forschungsprojekten oder Publikationsvorhaben, Statusgewinn oder -verlust, Bestätigung oder Infragestellung der eigenen Position etc.) können die Wahrnehmung und das Verhalten beeinflussen. Bisweilen werden direkte finanzielle und indirekte Interessenkonflikte unterschieden, welche mittelbar zu finanziellen Vorteilen führen können, etwa über Beförderungen (Schünemann et al. 2015). Relevant sind jeweils nur diejenigen Interessenkonflikte, die durch die betreffende Thematik oder Aufgabe berührt werden.

\section{Prinzipien}

Es gibt reichlich Hinweise in der Literatur, dass sich Interessenkonflikte auf das ärztliche

\section{Vielfach geschieht der Einfluss}

\section{von Interessenkonflikten}

auf das Urteilsvermögen unbewusst.

Verhalten (z.B. hinsichtlich Verschreibungsverhalten, Überweisungen etc.) auswirken können (siehe dazu Reinhart et al., 2009). Es ist auch bekannt, dass zahlreiche Vorsitzende oder Mitglieder von Leitlinien-Kommissionen Interessenkonflikte haben (Lenzer et al. 2013, Moynihan et al. 2013) und dass sich 
Interessenkonflikte auf die Empfehlungen auswirken können, wobei zu dieser Frage noch weiterer Forschungsbedarf besteht (Norris et al. 2011). Zugleich ist der Anspruch an die Leitlinienarbeit hoch, wie die GRADE Working Group formuliert hat: "Guideline panels should consider all the relevant factors (criteria) that influence a decision or recommendation in a structured, explicit, and transparent way and provide clinicians with clear and actionable recommendations» (Coelle-Alonso et al. 2016). Voreingenommenheit, die u.a. durch Interessenkonflikte hervorgerufen werden kann, gefährdet diese Arbeit erheblich. Von daher ist die Frage, welche Prinzipien den Umgang mit Interessenkonflikt prägen sollten, von grosser Relevanz.

Die üblicherweise ins Feld geführten Prinzipien betreffen die Offenlegung, das Vermeiden und das Management von Interessenkonflikten (Lo et al. 2009, Lieb et al. 2011). Als weitere, noch vorgängige Strategie ist die Sensibilisierung für die Problematik zu nennen. Dieser Sensibilisierung dienen z.B. die vorliegenden Empfehlungen.

Offenlegung: Gemäss dem Transparenzprinzip müssen Interessenkonflikte offengelegt werden. Dies gilt für individuelle Mitglieder ebenso wie für die Finanzierung der Leitlinienentwicklung. Üblicherweise unterstützen Formulare, welche gezielt relevante Kategorien abfragen, diesen Prozess. Bisweilen wird die Offenlegung auf finanzielle Zuwendungen eng geführt, womit andere, durchaus ebenfalls wirkmächtige Interessenkonflikte aus dem Blick geraten. Das Dokumentationsprinzip besagt, dass Leistungen schriftlich festzuhalten sind, so dass nachvollzogen werden kann, welcher Art die Zuwendung ist, welchen Zweck sie hat und welche Leistungen konkret erbracht werden (AWMF 2010).

Um die Offenlegung voranzutreiben, wurde in einem Beitrag im British Medical Journal der Vorschlag gemacht, Journals sollten Guidelines stets zusammen mit einem Guideline Panel Review publizieren. Dieser Review sollte sein Augenmerk auf folgende "red flags» legen: Der Sponsor ist eine Fachgesellschaft, die substantielles Funding von der Industrie erhält; der Sponsor ist ein Unternehmen oder unbekannt; der Vorsitzende der Kommission hat finanzielle Interessenkonflikte; es gibt Hinweise darauf, dass die Kommissionsmitglieder gezielt daraufhin ausgewählt worden sind, bestimmte Positionen zu vertreten; es wurden keine Methodologie-Experten beigezogen, um die Evidenz zu evaluieren; es gab keinen externen Review; es wurden keine nicht-ärztlichen Experten oder Patientenvertreter beigezogen (Lenzer et al. 2013).

Vermeiden: Hierunter fallen verschiedene Strategien. Zum einen können Interessenkon- flikte vor Antritt einer Leitlinien-Tätigkeit beendet werden, z.B. indem die Tätigkeit in einem Advisory Board aufgegeben wird («divestment»). Zum anderen können signifikante Interessenkonflikte zum Ausschluss ("prohibition/exclusion») aus bzw. zur Nichtaufnahme in eine Leitlinien-Kommission führen. Das Institute of Medicine geht sogar noch weiter: "Groups that develop clinical practice guidelines should generally exclude as panel members individuals with conflicts of interest and should not accept direct funding for clinical practice guideline development from medical product companies or company foundations.» Nur ausnahmsweise, wenn keine Alternativen existieren, sollen Mitglieder mit Interessenkonflikten aufgenommen werden dürfen; diese müssen jedoch in der Minderheit bleiben. Für die/den Vorsitzende/n werden jedoch keine Ausnahmen zugelassen (Lo et al. 2009). Mögliche Abstufung sind die Nichtaufnahme in die Kommission, aber das Beiziehen als externer Experte, oder die Enthaltung bei Abstimmungen.

\section{Ein klar definierter, reflektierter Um-} gang mit Interessenkonflikten kann somit einen signifikanten Beitrag zur Qualität einer Guideline leisten.

Management: Nachdem nicht immer vermeidbar ist, dass Mitwirkende an Leitlinienprozessen Interessenkonflikte haben, ist zu klären, wie Interessenkonflikte zu bewerten und wie mit ihnen umzugehen ist. Bei der Evaluation von Zuwendungen gelten das Trennungsprinzip - Zuwendungen müssen unabhängig von der Kommissionsarbeit und der inhaltlichen Positionierung sein - und das Äquivalenzprinzip, welches fordert, dass Leistung und Gegenleistung in einem angemessenen Verhältnis stehen (AWMF 2010). Beim Management von Interessenkonflikten in einer Kommission können protektive Faktoren gegen Bias genutzt werden, so etwa die interdisziplinäre/-professionelle $\mathrm{Zu}$ sammensetzung der Kommission mit einem Pluralismus von Stand-punkten; der systematische Bezug auf die verfügbare Evidenz; der Einsatz strukturierter Verfahren der Konsensfindung; der Einbezug unabhängiger Methodiker und Moderatoren sowie eine externe Begutachtung und/oder öffentliche Konsultation (Kopp 2015). Auf diese Weise kann es zumindest teilweise gelingen, interessenkonfliktbedingte Positionen einzubinden oder in Perspektive zu setzen.

Auch für die Erstellung bzw. die Evaluation von Interessenkonfliktregelungen können Prinzipien benannt werden (Lo et al. 2009): Das Prinzip der Proportionalität, welches nach der Effizienz der eingesetzten Strategien zum
Umgang mit Interessenkonflikten fragt; das Prinzip der Transparenz, welches die Verständlichkeit und Zugänglichkeit der Regelung einfordert; das Prinzip der Verantwortlichkeit, gemäss dem anzugeben ist, wer für die Umsetzung und Aktualisierung der Leitlinie zuständig ist; sowie das Prinzip der Fairness, gemäss dem die Regelung für alle gleichermassen gelten soll.

\section{Leitplanken}

Guidelines gelten vielfach als wichtige Orientierungshilfen in der klinischen Praxis. Wie die "Choosing Wisely»-Initiativen zeigen, können gerade Prozesse, welche von wissenschaftlichen Fachgesellschaften getragen werden, eine grosse Dynamik entfalten und Akzeptanz innerhalb der Ärzteschaft gewinnen. In der Schweiz werden häufig internationale Guidelines übernommen. Aber selbst dieser Prozess beinhaltet in aller Regel eine Adaptation an die Verhältnisse des Schweizerischen Gesundheitswesens und einen Konsentierungsprozess innerhalb der nationalen Fachgesellschaft. Die Entwicklung eines Rahmenwerks für Guidelines ist damit auch für die Schweiz interessant.

Der Umgang mit Interessenkonflikten ist ein Aspekt eines solchen Rahmenwerks zur Guideline-Entwicklung. Die nachfolgend aufgeführten Leitplanken (LP) gehen davon aus, dass sich Interessenkonflikte in der Guidelinearbeit nicht gänzlich vermeiden lassen und dass ein differenzierter Ansatz angezeigt ist, der möglichst viel Expertise einschliessen kann, ohne inhaltliche Verzerrungen durch Interessenkonflikte in Kauf nehmen zu müssen. Die Leitplanken sind mit den Prinzipien des Guidelines International Network (GIN) kompatibel (Schünemann et al. 2015).

\section{Bei der Guideline-Entwicklung sind die Perspektiven von Patienten und ihrem Umfeld zu berücksichtigen.}

LP1: Eine wichtige Voraussetzung für einen effektiven Umgang mit Interessenkonflikten, der sich nicht nur in Formalien erschöpft, ist eine Sensibilisierung für die Thematik. In Kommissionen finden sich typischerweise verschiedene, teils widersprüchliche Interessen. Problematisch sind jedoch Interessenkonflikte, die - besonders im Falle eines ungenügenden Managements und einer unausgewogenen Zusammensetzung der Gruppe - eine qualitativ hochstehende Guidelinearbeit ernsthaft gefährden können. Interessenkonflikte bringen ein Risiko für Voreingenommenheit mit sich, die der Selbstkorrektur nur bedingt zugänglich ist. Dieser «bias blind spot» führt dazu, dass die eigene Verzerrung der Wahrnehmung und 


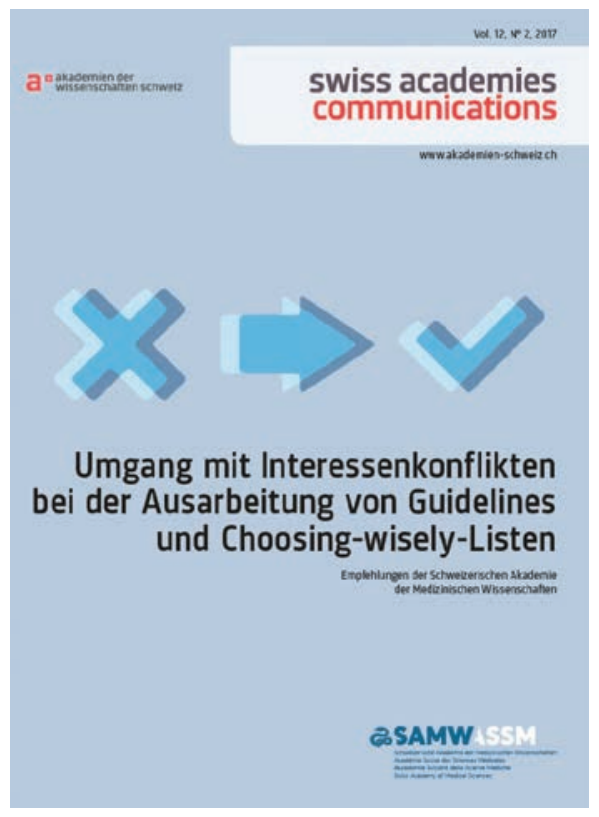

des Urteils oft nicht wahrgenommen oder unterschätzt wird.

LP2: Die Offenlegung von Interessen ist eine Frage der professionellen Integrität. Für die Offenlegung kommen zweckmässige Formulare zum Einsatz, welche verschiedene Typen von Interessen gezielt abfragen. Bestehen innerhalb der Guideline-Kommission Interessenkonflikte, sind diese innerhalb der Gruppe zur Kenntnis zu bringen, ebenso wie auch Änderungen, etwa neu auftretende Interessenbindungen. Die Interessenerklärungen sind zusammen mit der Guideline, den Autoren und einer Dokumentation des Entstehungsprozesses in systematischer Form zugänglich zu machen.

LP3: An der Erstellung von Guidelines wirken Personen mit, welche keine relevanten Interessenkonflikte haben, die die Kommissions- arbeit beeinträchtigen können. Wichtig ist auch die ausgewogene Zusammensetzung der gesamten Gruppe. Die Beurteilung von Interessenkonflikten hat jedoch mit Augenmass zu erfolgen: Nicht alle Interessenkonflikte sind für die Arbeit an einer Leitlinie relevant, und manche sind so geringfügig, dass sie nicht notwendigerweise zu einem Ausschluss aus der Kommissionsarbeit führen müssen. Es bietet sich ein abgestuftes System an, welches für den Vorsitz bzw. dessen Stellvertretung keine Interessenkonflikte zulässt, bei Mitgliedern mit moderaten Interessenkonflikten eine Einschränkung des Stimmrechts vorsieht und Personen mit gravierenden Interessenkonflikten bei Bedarf als externe Experten beizieht. Durch wen und wie die Beurteilung erfolgt und wie ein allfälliger Rekurs geregelt ist, muss von der betreffenden Organisation in transparenter Weise festgelegt werden.

LP4: Der Guidelineprozess wird von einer unabhängigen Institution getragen. In jedem Fall darf die Finanzierung der Guideline-Entwicklung selbst nicht zu Interessenkonflikten führen. Eine Registrierungsstelle zur Anmeldung der Arbeiten, ein externer Review vor der Publikation oder ein Oversight Committee unterstützen die Einhaltung der Interessenkonfliktregelung.

LP5: Der Umgang mit Interessenkonflikten erfordert ein aktives Management. Einer von Interessenkonflikten unbelasteten Leitung bzw. Moderation der Guideline-Kommission kommt daher besondere Bedeutung zu. So ist zum Beispiel über den Ausschluss individueller Mitglieder von Bewertungen oder Abstimmungen zu entscheiden. Die Ausstandsregeln sind im Vorfeld transparent festzulegen. $\mathrm{Zu}$ gleich sind protektive Faktoren, wie etwa das Rückbinden der Diskussion an die Evidenzlage oder eine strukturierte Konsensfindung, zu nutzen.
LP6: Bei der Guideline-Entwicklung sind die Perspektiven von Patienten und ihrem Umfeld zu berücksichtigen. Wenn Patientenvertreter in Kommissionen Einsitz haben, ist eine Prüfung von Interessenkonflikten wie

\section{Guidelines gelten vielfach als} wichtige Orientierungshilfen in der klinischen Praxis.

bei allen anderen Kommissionsmitgliedern vorzunehmen. Zu erwägen ist auch die Erstellung von Patientenversionen der Guidelines, um den Zugang für Laien zu verbessern.

LP7: Es wird die Möglichkeit zur Kommentierung der Guideline im Sinne eines post-publication review eingeräumt. Guidelines sind periodisch zu aktualisieren. Da nicht nur die Entwicklung von Guidelines, sondern auch deren Disseminierung und Implementierung von Interessenkonflikten belastet sein kann, wäre eine Evaluation dieser Phasen zumindest bei Guidelines mit hohem erwarteten Impact wünschenswert.

\section{Literatur}

Die Broschüre und die Online-Version enthalten eine ausführliche Literaturliste.

Bildnachweis

(c) SAMW

Korrespondenz:
SAMW
Haus der Akademien
Laupenstrasse 1
CH-3001 Bern
mail[at]samw.ch

